\title{
LncRNA-KCNQ10T1 Promotes the Odontoblastic Differentiation of Dental Pulp Stem Cells Via Regulating miR-153-3p/RUNX2 Axis
}

\section{Xiaohui Lu}

Affiliated Hospital of Nantong University

Jiawen Zhang

Affiliated Hospital of Nantong University

\section{Yuanzhou Lu}

affiliated tongzhou hospital of nantong uiversity

Jing Xing

Affiliated Hospital of Nantong University

Min Lian

Affiliated Hospital of Nantong University

\section{Guijuan Feng}

Affiliated Hospital of Nantong University

\section{Dan Huang}

Affiliated Hospital of Nantong University

\section{Chenfei Wang}

Affiliated Hospital of Nantong University

\section{Nimei Shen}

second affiliated hospital of nantong university

xingmei feng ( $\nabla$ xingmeifeng@ntu.edu.cn )

Affiliated Hospital of Nantong University

\section{Research}

Keywords: LncRNA-KCNQ10T1, odontoblastic differentiation, dental pulp stem cells, miR-153-3p, RUNX2

Posted Date: October 5th, 2021

DOl: https://doi.org/10.21203/rs.3.rs-942120/v1

License: (c) (1) This work is licensed under a Creative Commons Attribution 4.0 International License.

Read Full License 


\section{Abstract}

Background and Objective: Long non-coding RNAs (LncRNAs) play a key role in the odontoblastic differentiation. This study aimed to explore the role of LncRNA-KCNQ10T1 in the odontoblastic differentiation of human dental pulp stem cells (DPSCs) and its possible mechanism.

Methods: The expression of LncRNA-KCNQ10T1, miR-153-3p, RUNX2 in the odontoblastic differentiation was detected by qRT-PCR. Interaction between LncRNA-KCNQ10T1 and miR-153-3p and interaction between miR-153-3p and RUNX2 were detected by dual-luciferase assay. The cell viability of DPSCs was detected by cell counting kit-8 (CCK-8), and the effect of LncRNA-KCNQ10T1 and miR-153-3p on the odontoblastic differentiation of DPSCs was observed by alizarin red staining, alkaline phosphatase (ALP) activity assay and Western blot for RUNX2, DSPP, DMP-1.

Results: During odontoblastic differentiation of DPSCs, the expression of LncRNA-KCNQ10T1 increased, miR-153-3p expression decreased, and RUNX2 expression increased. Dual-luciferase assay showed that LncRNA-KCNQ10T1 sponges miR-153-3p and miR-153-3p targets on RUNX2. After LncRNA-KCNQ10T1 and miR-153-3p expressions of DPSCs were changed, the cell viability was not notably changed, but the odontoblastic differentiation was notably changed which was confirmed with alizarin red staining, ALP activity and Western blot for RUNX2, DSPP, DMP-1.

Conclusion: LncRNA-KCNQ10T1 promotes the odontoblastic differentiation of DPSCs via regulating miR153-3p/RUNX2 axis, which may provide a therapeutic clue for odontogenesis.

\section{Background}

Human dental pulp stem cells (DPSCs) are a kind of adult stem cells with high proliferation, self-renewal ability and multi-differentiation potential, which are mainly derived from dental pulp tissue. DPSCs can not only differentiate into dentin, but also into bone, cartilage, fat, myogenic and other mesodermalderived cells $(1,2)$. Human DPSCs are usually taken from extracted third molars or healthy premolars that need to be extracted due to orthodontics. They are easier to obtain and have less ethical pressure. Therefore, DPSCs are ideal seed cells for tissue engineering(3-5). The ability of DPSCs to differentiate into odontoblasts plays an important role in maintaining the dynamic balance of dental pulp tissue and tooth regeneration, and is the basis for future use of tissue engineering to achieve dentin regeneration(68). Odontoblastic differentiation of DPSCs involves many factors such as biological scaffolds, regulatory genes and signal pathways(8-12). MicroRNAs (miRNAs, miRs) as post-transcriptional inhibitors, recognize and bind to the 3 'untranslated region (3'UTR) of the target gene to inhibit the translation of the target gene protein, and have complex regulatory effects on the body's physiological/pathological activities, including the process of odontoblastic differentiation(13-15). Long non-coding RNAs (LncRNAs) as competing endogenous RNAs (ceRNAs) play key role in cell cycle, migration, proliferation, differentiation and apoptosis through sponging miRNAs to regulate miRNA targets. More and more 
studies have shown that LncRNAs participate in the differentiation process of odontoblasts through sponging miRNAs $(10,16,17)$.

Studies have shown that runt-related transcription factor 2 (RUNX2) plays a key role in the odontoblastic differentiation. Its expression promotes the odontoblastic differentiation(18-20). In this study, the software targetscan (http://www.targetscan.org) was used to analyze which miRNAs maybe target the RUNX2, and the result showed RUNX2 may be targeted by miR-153-3p. We used LncBase Predicted v. 2 database (http://carolina.imis. athena-innovation.gr) to analyze the potential LncRNAs which interact with miR-153-3p, and the results showed LncRNA-KCNQ10T1 contains potential binding site of miR-153-3p. Jiang et al reported that miR-153-3p inhibited osteogenic differentiation of periodontal ligament stem cells via targeting KDM6A and regulating the ALP, RUNX2 and OPN transcription(21). Studies showed LncRNA-KCNQ10T1 regulated osteogenic differentiation by sponging miR-214(22), miR-320a(23). Yu et al reported that knockdown of IncRNA-KCNQ10T1 in tendon stem cell inhibited the osteogenic differentiation by regulating miR-138/PPARY or RUNX2 axis(24). However, the role of IncRNA-KCNQ10T1 and miR-153-3p in the differentiation of DPSCs into odontoblasts is still unclear. The study aimed to explore the effect of IncRNA-KCNQ10T1 and miR-153-3p on the odontoblastic differentiation of DPSCs and confirm its regulation axis.

\section{Methods}

\section{DPSCs culture}

This study was approved by the Ethics Committee of the Affiliated Hospital of Nantong University. The cell culture and identification was performed as described in our previous studies(13). Briefly, the pulp from normal human impacted third molars was collected and digested with $3 \mathrm{mg} / \mathrm{ml}$ collagenase type I for $1 \mathrm{~h}$ at $37^{\circ} \mathrm{C}$. Donor of the impacted third molar had given informed consent. Single-cell suspensions of dental pulp were cultured and passaged in Dulbecco modified Eagle medium (DMEM) supplemented with $10 \%$ fetal bovine serum (FBS), $100 \mathrm{U} / \mathrm{mL}$ penicillin and $100 \mu \mathrm{g} / \mathrm{mL}$ streptomycin at $37^{\circ} \mathrm{C}$ under $5 \% \mathrm{CO}_{2}$. The fourth passage cells were used in the following experiments.

\section{Cell transfection}

DPSCs were cultured in a 6-well culture plate at a concentration of $5 \times 10^{3} / \mathrm{ml}$. After $24 \mathrm{~h}$, they were transfected with KCNQ10T1 siRNA, siRNA-NC (RuiboBio, China), overexpression vectors of KCNQ10T1 (pcDNA-KCNQ10T1) (Sangon Biotech, China), miR-153-3p inhibitor, inhibitor-NC, miR-153-3p mimic and mimic NC (ThemoFisher, USA) with lipofectamine 2000 (ThemoFisher, USA) according to the transfection instructions, the cells were incubated in $5 \% \mathrm{CO}_{2}, 37^{\circ} \mathrm{C}$ incubator and a blank control group was set up. After 48 hours, the transfected cells were taken for the following experiment.

\section{Cell viability assay}


The cell counting Kit-8 (CCK-8; Beyotime Biotechnology, China) was used to detect the viability of the DPSCs according to the manufacturer instructions, and the OD value was read at $450 \mathrm{~nm}$.

\section{Odontoblastic differentiation culture}

The odontoblastic differentiation culture was performed as described in our previous study(13). Briefly, the fourth passages DPSCs were cultured in odontogenic differentiation medium containing a minimum essential medium (Invitrogen, Carlsbad, CA), $50 \mathrm{mg} / \mathrm{mL}$ a-ascorbic acid, 15\% FBS, $10 \mathrm{mmol} / \mathrm{L} \mathrm{b}$ glycerophosphate, $10 \mathrm{nmol} / \mathrm{L}$ dexamethasone (Sigma-Aldrich, St Louis, M0), $0.292 \mathrm{mg} / \mathrm{mL}$ glutamine, $100 \mu \mathrm{g} / \mathrm{mL}$ streptomycin, and $100 \mathrm{U} / \mathrm{mL}$ penicillin for 14 days.

\section{Quantitative real-time PCR (qRT-PCR)}

The total RNA in cells was extracted using Trizol (Invitrogen, USA), and quantified with agarose gel (Sigma-Aldrich, USA). For LncRNA and mRNA, cDNA was synthesized using TransScript First-Strand cDNA Synthesis SuperMix (Transgen, China). For miRNA, cDNA was synthesized using TransScript miRNA First-Strand cDNA Synthesis SuperMix (Transgen, China). GAPDH and U6 were used as the internal reference. The gene expression level was calculated with $2^{-} \Delta \Delta \mathrm{Ct}$ method. The primer sequences were shown in the Table-1.

\section{Luciferase reporter gene experiment}

The wild-type (WT) binding fragment of IncRNA-KCNQ10T1 or WT core sequence of 3'UTR of RUNX2 was cloned into pMIR-Report luciferase vector (Promega Corporation, USA) to construct WT-IncRNAKCNQ10T1 reporter vector or WT-RUNX2 reporter vector. The fragment of IncRNA-KCNQ10T1 or core sequence of 3'UTR of RUNX2 were mutated (MU) and cloned into pMIR-Report luciferase vector to obtain MU-IncRNA-KCNQ10T1 reporter vector or MU-RUNX2 reporter vector. The WT-IncRNA-KCNQ10T1, MUIncRNA-KCNQ10T1 and miR-153-3p mimic (ThemoFisher, USA) or NC were co-transfected into HEK293 cells to investigate the relationship between IncRNA-KCNQ10T1 and miR-153-3p. The WT-RUNX2, MURUNX2 and miR-153-3p mimic (ThemoFisher, USA) or NC were co-transfected into HEK293 cells to investigate the relationship between miR-153-3p and RUNX2. After transfection $48 \mathrm{~h}$, relative luciferase activity was analyzed with a dual-luciferase reporter assay system (Promega Corporation, USA) according to the manufacturer instructions.

\section{Alkaline phosphatase (ALP) activity assay}

After 14 days of odontoblastic differentiation, the cells were lysed with $1 \%$ Triton X-100 for 15 min, and centrifuged at 10,000 $\mathrm{g}$ for $5 \mathrm{~min}$, then the supernatant was collected and detected with ALP Assay Kit (Beyotime Biotechnology, China) according to the manufacturer instructions, and the OD value was read at $405 \mathrm{~nm}$.

\section{Alizarin red staining}

After 14 days of odontoblastic differentiation, the cells were incubated with $2 \%$ alizarin red staining solution (Beyotime, China) for $10 \mathrm{~min}$ at room temperature. Then the cells were observed under an 
inverted microscope and the cell mineralization was quantified with alizarin red extracted with $100 \mathrm{mM}$ cetylpyridinium chloride solution (Sigma, USA), and the OD value was read at $570 \mathrm{~nm}$.

\section{Western blot analysis}

After 14 days of odontoblastic differentiation, the total protein of cells was extracted by RIPA buffer (Beyotime, China). The protein was electrophoresed and transferred to PVDF membrane. After PVDF membrane blocked with $5 \%$ BSA, it was successively incubated with the primary antibody: rabbit antiRUNX2 primary antibody (1:800, Abcam, UK), rabbit anti-DSPP (1:1000, Abcam), rabbit anti-DMP-1 (1:1000, Abcam), or mouse anti- $\beta$-actin (1:1000, Abcam, UK) and the second antibody: IRDye 700conjugated affinity-purified goat anti-mouse (1:4,000, Rockland Immunochemicals, USA) or IRDye 800conjugated affinity-purified goat anti-rabbit second antibody (1:4,000, Rockland Immunochemicals, USA). The relative protein expression level was analyzed with Odyssey laser scanning system (LI-COR Inc., USA).

\section{Statistical analysis}

The SPSS 22.0 software was used to analyze the data of this research. The data of this study were presented as mean \pm standard deviation $(M \pm S D)$. Comparison among groups was tested with independent $t$ test or one-way analysis of variance (ANOVA) followed by Tukey's test. The Pearson analysis was used to analyze the correlation. Statistical graph was made using GraphPad Prism 7 software. $P<0.05$ was considered statistically significant.

\section{Results}

\section{LncRNA-KCNQ10T1, miR-153-3p and RUNX2 expression levels during odontoblastic differentiation}

During the odontoblastic differentiation of DPSCs, the expressions of LnCRNA-KCNQ10T1, miR-153-3p and RUNX2 were detected at $0,1,3,7,14 \mathrm{~d}$. The relative expression levels of LncRNA-KCNQ10T1, miR153-3p and RUNX2 were quantified with the expression level at $0 \mathrm{~d}$ when the DPSCs did not begin the odontoblastic differentiation. On the $1 \mathrm{~d}$ of differentiation of odontoblasts, the expressions of LncRNAKCNQ10T1 and RUNX2 increased, and miR-153-3p expression level decreased, and they reached a peak at $3 d$, then their expression levels at 7, $14 d$ was similar to that at $3 d$ (Fig. 1A-C). Spearman correlation analysis showed that LnCRNA-KCNQ10T1 and RUNX2 expression levels were positively correlated; LncRNA-KCNQ10T1 and miR-153-3p expression levels were negatively correlated; miR-153-3p and RUNX2 expression levels were negatively correlated (Fig. 1D-F).

\section{The effect of LncRNA-KCNQ10T1 on the cell viability and odontoblastic differentiation of DPSCs}

After transfection, the LncRNA-KCNQ10T1 expression level was detected by qRT-PCR. LncRNAKCNQ10T1 expression level of DPSCs transfected with pCDNA-KCNQ10T1 significantly increased, and 
that of DPSCs transfected with KCNQ10T1 siRNA notably decreased (Fig. 2A). The viability of DPSCs was assessed with CCK-8 kit. Compared with control, the differences of OD value among groups were not statistically significant. The transfection did not affect the viability of DPSCs (Fig. 2B).

After 14 days of culture, the odontoblastic differentiation of DPSCs was detected by ALP activity, Alizarin red staining and Western blot for RUNX2, DSPP, and DMP-1. The result of ALP activity assay showed the OD value of the pcDNA-KCNQ10T1 group was higher than that of control and NC groups, while the OD value of the KCNQ10T1 siRNA group was lower than that of control and NC groups (Fig. 2C). The result of Alizarin red staining displayed that mineralized bone matrix increased in the pcDNA-KCNQ10T1 group and decreased in the KCNQ10T1 siRNA group comparison with the control and NC groups (Fig. 2D). The result of Western blot showed the protein expression levels of RUNX2, DSPP, and DMP-1 in the pcDNAKCNQ10T1 group increased and in the KCNQ10T1 siRNA group decreased compared with the control and NC groups (Fig. 2E).

\section{Overexpression of miR-153-3p reverses the promotion of odontoblastic differentiation induced by LncRNA- KCNQ10T1}

After transfection, LncRNA-KCNQ10T1 and miR-153-3p expression levels were detected by qRT-PCR. LncRNA-KCNQ10T1 expression level of DPSCs transfected with pcDNA-KCNQ10T1 or pcDNAKCNQ10T1 + mimic or pcDNA-KCNQ10T1 + mimic NC significantly increased (Fig. 3A), and miR-153-3p expression level of DPSCs transfected pcDNA-KCNQ10T1 or pcDNA-KCNQ10T1 + mimic NC significantly decreased, and miR-153-3p expression level of DPSCs transfected with pcDNA-KCNQ10T1 + mimic was similar to the control group (Fig. 3B). In addition, the results of CCK-8 assay showed the transfection did not affect the viability of DPSCs (Fig. 3C).

After 14 days of odontoblastic differentiation, the mineralized bone matrix, ALP activity and protein expression levels of RUNX2, DSPP and DMP-1 in the pcDNA-KCNQ10T1 group increased comparison with the control group. While DPSCs were co-transfected with pcDNA-KCNQ10T1 and miR-153-3p mimic, the mineralized bone matrix, ALP activity, RUNX2, DSPP, and DMP-1 protein expression levels were close to the control group. When DPSCs were co-transfected with pCDNA-KCNQ10T1 and mimic NC, the mineralized bone matrix, ALP activity, RUNX2, DSPP, and DMP-1 protein expression levels were similar to the pcDNA-KCNQ10T1 group (Fig. 3D-F).

\section{LncRNA-KCNQ10T1 act as a sponge of miR-153-3p and miR-153-3p target on RUNX2}

Luciferase reporter gene experiment was used to verify that LncRNA-KCNQ10T1 act as a sponge of miR153-3p and miR-153-3p target on RUNX2. The result showed that when the binding fragment of LncRNAKCNQ10T1 or core sequence of 3'UTR of RUNX2 was mutated, the luciferase relative activity of mimic group was similar to the NC group. However, in the WT-LncRNA-KCNQ10T1 or WT-RUNX2 reporter gene system, the relative activity of luciferase in mimic group was significantly lower than that in NC group. 


\section{Discussion}

Odontoblasts are terminally differentiated cells from DPSCs and are one of the main cells that form dental tissues. Odontoblastic differentiation is the prerequisite for dentin formation. Studying the influence of various signaling pathways on the differentiation of odontoblasts, regulating the expression of various signaling pathways, and promoting the differentiation of odontoblasts will be of great significance for the treatment of various dentin-related diseases. More and more researches prove that LncRNAs and miRNAs play key roles in odontoblastic differentiation $(10,16,17)$. In this study, we found that LnCRNA-KCNQ10T1 promoted the odontoblastic differentiation of DPSCs via regulating miR-1533p/RUNX2 axis.

RUNX2 is one of the members of the Runt family, which is an specific transcription factor of odontoblastic differentiation of DPSCs(18). In this study, during the odontoblastic differentiation of DPSCs, the expression of RUNX2 increased, which is consistent with the research results of other scholars(25). Studies showed many miRNAs regulated the odontoblastic differentiation via targeting on RUNX2 $(15,26,27)$. In this study, we used targetscan software to found miR-153-3p have the binding site of RUNX2 3'UTR. Jiang et al reported that miR-153-3p inhibited osteogenic differentiation of periodontal ligament stem cells via targeting KDM6A and regulating the ALP, RUNX2 and OPN transcription(21). The odontoblastic differentiation process is similar to the osteogenic differentiation. Therefore, in this study, the expression level of miR-153-3p during odontoblastic differentiation of DPSCs was detected and the result showed it decreased in the process and miR-153-3p expression level was negatively correlated with RUNX2 expression level. The luciferase reporter gene experiment confirmed that RUNX2 is a target of miR153-3p. The results indicated that miR-153-3p was indeed involved in the odontoblastic differentiation of DPSCs, and it was a negative regulatory factor.

The mechanism by which the expression of miR-153-3p decreases during the differentiation of odontoblasts is still unclear. LncRNAs as ceRNAs can sponge miRNAs. We used LncBase Predicted v.2 database to found LncRNA-KCNQ10T1 contained the potential binding site of miR-153-3p. Study showed that knockdown of LnCRNA-KCNQ10T1 in tendon stem cell inhibited the osteogenic differentiation(24). The result of qRT-PCR in this study showed LncRNA-KCNQ10T1 increased during odontoblastic differentiation of DPSCs and its expression level was negatively correlated with miR-153-3p. The luciferase reporter gene experiment confirmed that LncRNA-KCNQ10T1 sponges miR-153-3p. The results indicated that LnCRNA-KCNQ10T1 positively regulated the odontoblastic differentiation of DPSCs. Therefore, we constructed pcDNA-KCNQ10T1 or KCNQ10T1 siRNA to transfect DPSCs to enhance or downregulate LncRNA-KCNQ10T1 expression level. The result of CCK-8 showed LncRNA-KCNQ10T1 expression changes had no effect on the cell viability. The odontoblastic differentiation of DPSCs was detected by alizarin red staining, ALP activity and Western blot for RUNX2, DSPP and DMP-1, and the findings showed down-regulated LnCRNA-KCNQ10T1 expression inhibited the odontoblastic differentiation of DPSCs, while LnCRNA-KCNQ10T1 overexpression promoted the odontoblastic differentiation of DPSCs. When DPSCs were co-transfected with pcDNA-KCNQ10T1 and miR-153-3p 
mimic, DPSCs overexpressed LncRNA-KCNQ10T1 and miR-153-3p at the same time, LncRNAKCNQ10T1's promotion of odontoblast differentiation was reversed.

In summary, our data demonstrated that LnCRNA-KCNQ10T1 promotes the odontoblastic differentiation of DPSCs via regulating miR-153-3p/RUNX2 axis, which may provides a therapeutic clue for odontogenesis.

\section{Declarations}

\section{Ethics approval and consent to participate}

The study was approved by the Ethics Committee of the Affiliated Hospital of Nantong University.

\section{Consent for publication}

Not applicable.

\section{Availability of data and materials}

The datasets used and/or analysed during the current study are available from the corresponding author on reasonable request.

\section{Competing interests}

The authors declare that they have no competing interests

\section{Funding}

This work was supported by National Natural Science Foundation of China (81500809) and Nantong Science Plan guiding project (MSZ19167).

\section{Authors' contributions}

GF and XF conceived and designed the experiments. $\mathrm{XL}, \mathrm{XC}, \mathrm{JX}, \mathrm{ML}, \mathrm{DH}$ and $\mathrm{YL}$ performed the experiments. $\mathrm{XL}$ and $\mathrm{XF}$ analyzed the data. $\mathrm{XL}$ and $\mathrm{XC}$ wrote the paper. All authors read and approved the final manuscript.

\section{Acknowledgements}

Not applicable.

\section{References}

1. Aydin S, Sahin F. Stem Cells Derived from Dental Tissues. Adv Exp Med Biol. 2019;1144:123-32. 
2. Nuti N, Corallo C, Chan BM, Ferrari M, Gerami-Naini B. Multipotent Differentiation of Human Dental Pulp Stem Cells: a Literature Review. Stem cell reviews reports. 2016;12(5):511-23.

3. Tatullo M, Marrelli M, Shakesheff KM, White LJ. Dental pulp stem cells: function, isolation and applications in regenerative medicine. J Tissue Eng Regen Med. 2015;9(11):1205-16.

4. Itoh Y, Sasaki Jl, Hashimoto M, Katata C, Hayashi M, Imazato S. Pulp Regeneration by 3-dimensional Dental Pulp Stem Cell Constructs. Journal of dental research. 2018;97(10):1137-43.

5. Dong Q, Wang Y, Mohabatpour F, Zheng L, Papagerakis S, Chen D, et al. Dental Pulp Stem Cells: Isolation, Characterization, Expansion, and Odontoblast Differentiation for Tissue Engineering. Methods in molecular biology. 2019;1922:91-101.

6. Moonesi Rad R, Atila D, Akgun EE, Evis Z, Keskin D, Tezcaner A. Evaluation of human dental pulp stem cells behavior on a novel nanobiocomposite scaffold prepared for regenerative endodontics. Materials science \& engineering C, Materials for biological applications. 2019;100:928 - 48.

7. Zhai Q, Dong Z, Wang W, Li B, Jin Y. Dental stem cell and dental tissue regeneration. Frontiers of medicine. 2019;13(2):152-9.

8. Moussa DG, Aparicio C. Present and future of tissue engineering scaffolds for dentin-pulp complex regeneration. J Tissue Eng Regen Med. 2019;13(1):58-75.

9. Cui D, Xiao J, Zhou Y, Zhou X, Liu Y, Peng Y, et al. Epiregulin enhances odontoblastic differentiation of dental pulp stem cells via activating MAPK signalling pathway. Cell proliferation. 2019;52(6):e12680.

10. Zhong J, Tu X, Kong Y, Guo L, Li B, Zhong W, et al. LncRNA H19 promotes odontoblastic differentiation of human dental pulp stem cells by regulating miR-140-5p and BMP-2/FGF9. Stem Cell Res Ther. 2020;11(1):202.

11. Zhang X, Ning T, Wang H, Xu S, Yu H, Luo X, et al. Stathmin regulates the proliferation and odontoblastic/osteogenic differentiation of human dental pulp stem cells through Wnt/beta-catenin signaling pathway. Journal of proteomics. 2019;202:103364.

12. Yu H, Zhang X, Song W, Pan T, Wang H, Ning T, et al. Effects of 3-dimensional Bioprinting Alginate/Gelatin Hydrogel Scaffold Extract on Proliferation and Differentiation of Human Dental Pulp Stem Cells. J Endod. 2019;45(6):706-15.

13. Lu X, Chen X, Xing J, Lian M, Huang D, Lu Y, et al. miR-140-5p regulates the odontoblastic differentiation of dental pulp stem cells via the Wnt1/beta-catenin signaling pathway. Stem Cell Res Ther. 2019;10(1):226.

14. Lin C, Zhang Q, Yu S, Lin Y, Li S, Liu H, et al. miR-3065-5p regulates mouse odontoblastic differentiation partially through bone morphogenetic protein receptor type II. Biochem Biophys Res Commun. 2018;495(1):493-8.

15. Li Q, Huang L. MiR-148a-3p Regulates the Invasion and Odontoblastic Differentiation of Human Dental Pulp Stem Cells via the Wnt1/beta-Catenin Pathway. International journal of stem cells. 2021.

16. Chen Z, Zhang K, Qiu W, Luo Y, Pan Y, Li J, et al. Genome-wide identification of long noncoding RNAs and their competing endogenous RNA networks involved in the odontogenic differentiation of human dental pulp stem cells. Stem Cell Res Ther. 2020;11(1):114. 
17. Fang F, Zhang K, Chen Z, Wu B. Noncoding RNAs: new insights into the odontogenic differentiation of dental tissue-derived mesenchymal stem cells. Stem Cell Res Ther. 2019;10(1):297.

18. Camilleri S, McDonald F. Runx2 and dental development. Eur J Oral Sci. 2006;114(5):361-73.

19. Han N, Zheng Y, Li R, Li X, Zhou M, Niu Y, et al. beta-catenin enhances odontoblastic differentiation of dental pulp cells through activation of Runx2. PloS one. 2014;9(2):e88890.

20. Qin X, Jiang Q, Komori H, Sakane C, Fukuyama R, Matsuo Y, et al. Runt-related transcription factor-2 (Runx2) is required for bone matrix protein gene expression in committed osteoblasts in mice. Journal of bone and mineral research: the official journal of the American Society for Bone and Mineral Research. 2021.

21. Jiang H, Jia P. MiR-153-3p inhibits osteogenic differentiation of periodontal ligament stem cells through KDM6A-induced demethylation of H3K27me3. J Periodontal Res. 2021;56(2):379-87.

22. Wang CG, Liao Z, Xiao H, Liu H, Hu YH, Liao QD, et al. LncRNA KCNQ10T1 promoted BMP2 expression to regulate osteogenic differentiation by sponging miRNA-214. Exp Mol Pathol. 2019;107:77-84.

23. Wang JL, Wei X, Wang AG, Bai Y, Wu XJ. KCNQ10T1 regulates osteogenic differentiation of hBMSC by miR-320a/Smad5 axis. Eur Rev Med Pharmacol Sci. 2020;24(6):2843-54.

24. Yu Y, Chen Y, Zhang X, Lu X, Hong J, Guo X, et al. Knockdown of IncRNA KCNQ10T1 suppresses the adipogenic and osteogenic differentiation of tendon stem cell via downregulating miR-138 target genes PPARgamma and RUNX2. Cell cycle. 2018;17(19-20):2374-85.

25. Tu S, Wu J, Chen L, Tian Y, Qin W, Huang S, et al. LncRNA CALB2 sponges miR-30b-3p to promote odontoblast differentiation of human dental pulp stem cells via up-regulating RUNX2. Cellular signalling. 2020;73:109695.

26. Sun Q, Liu H, Lin H, Yuan G, Zhang L, Chen Z. MicroRNA-338-3p promotes differentiation of mDPC6T into odontoblast-like cells by targeting Runx2. Molecular cellular biochemistry. 2013;377(1-2):1439.

27. Zhan FL, Liu XY, Wang XB. The Role of MicroRNA-143-5p in the Differentiation of Dental Pulp Stem Cells into Odontoblasts by Targeting Runx2 via the OPG/RANKL Signaling Pathway. Journal of cellular biochemistry. 2018;119(1):536-46.

\section{Tables}


Table-1 The primer sequences for qRT-PCR

\begin{tabular}{cl}
\hline Gene & Primer sequences $\left(5^{\prime}-3^{\prime}\right)$ \\
\hline \multirow{2}{*}{ lncRNA-KCNQ1OT1 } & Forward: ACTCACTCACTCACTCACT \\
& Reverse: CTGGCTCCTTCTATCACATT \\
miR-153-3p & Forward: ACACTCCAGCTGGGTTGCATAGTCACAAA \\
& Reverse: CAGTGCGTGTCGTGGAGT \\
RUNX2 & Forward: TGCCACCTCTGACTTCTGC \\
& Reverse: GATGAAATGCCTGGGAACTG \\
& Forward: GTGCTCGCTTCGGCAGCACAT \\
& Reverse: TACCTTGCGAAGTGCTTAAAC \\
& Forward: AGGTGAAGGTCGGAGTCAAC \\
GAPDH & Reverse: CGCTCCTGGAAGATGGTGAT \\
\end{tabular}

\section{Figures}
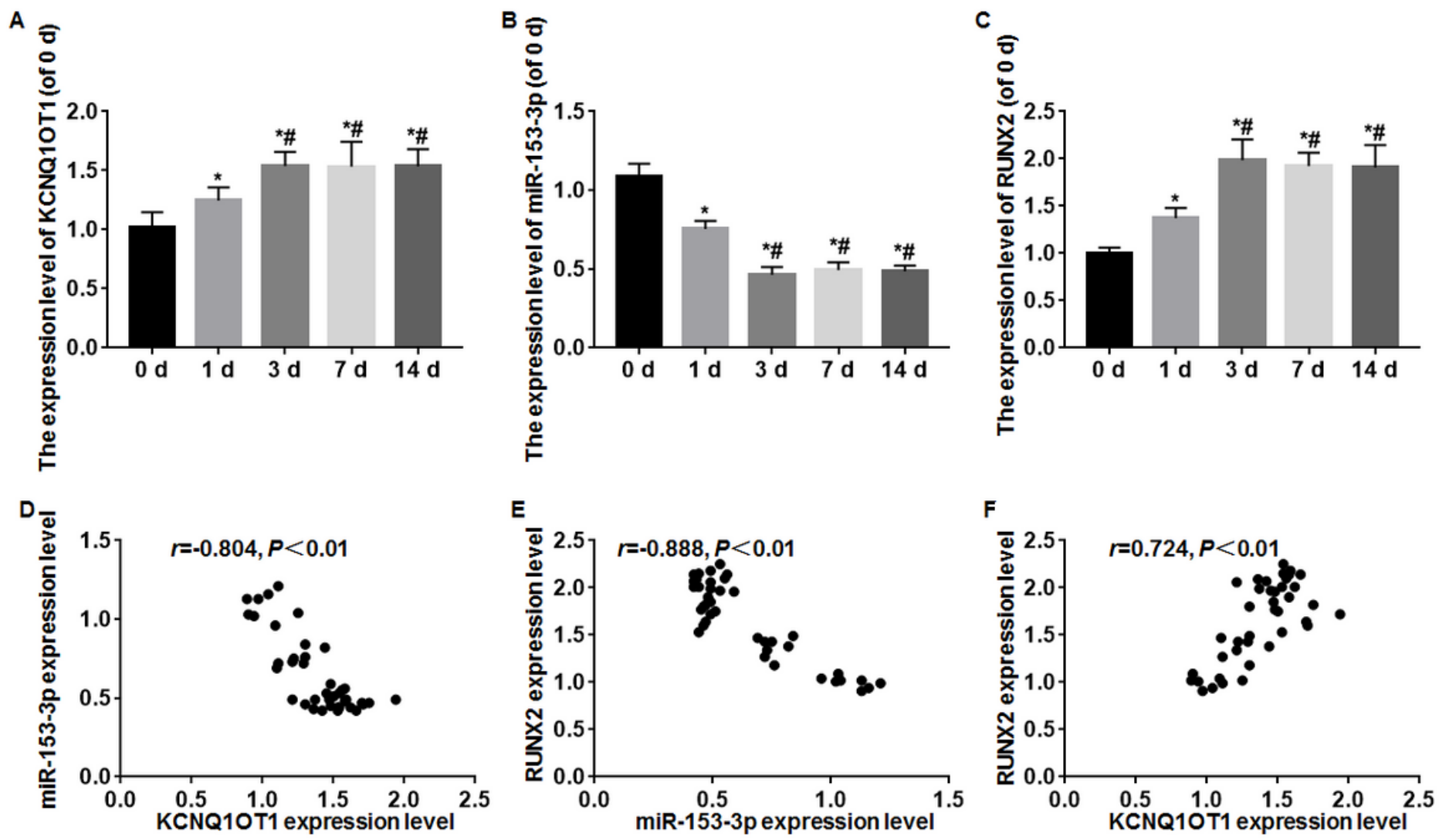

Figure 1 
LncRNA-KCNQ10T1, miR-153-3p and RUNX2 expression levels during odontoblastic differentiation were detected by qRT-PCR. (A) On the $1 \mathrm{~d}$ of differentiation of odontoblasts, the expression of LncRNAKCNQ10T1 increased and reached a peak at $3 \mathrm{~d}$. (B) On the $1 \mathrm{~d}$ of differentiation of odontoblasts, miR153-3p expression level decreased and reached a peak at $3 \mathrm{~d}$. (C) On the $1 \mathrm{~d}$ of differentiation of odontoblasts, the expression of RUNX2 increased and reached a peak at $3 \mathrm{~d}$. (D) LncRNA-KCNQ10T1 expression level was negatively correlated with miR-153-3p expression level. (E) miR-153-3p expression level were negatively correlated with RUNX2 expression level. (F) LncRNA-KCNQ10T1 expression level was positively correlated with RUNX2 expression level. * vs. 0 d, $\mathrm{P}<0.05$; \# vs. $1 \mathrm{~d}, \mathrm{P}<0.05$. 
A

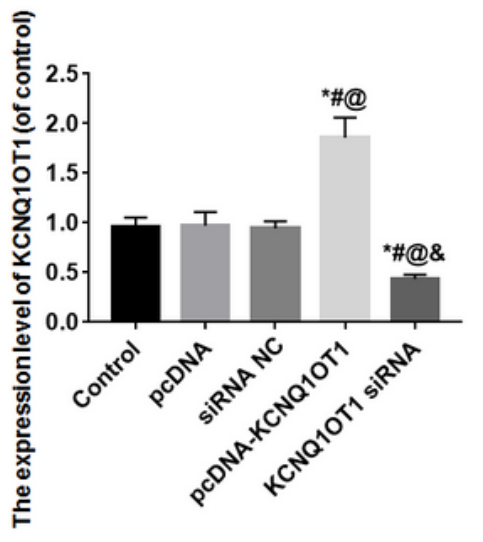

D
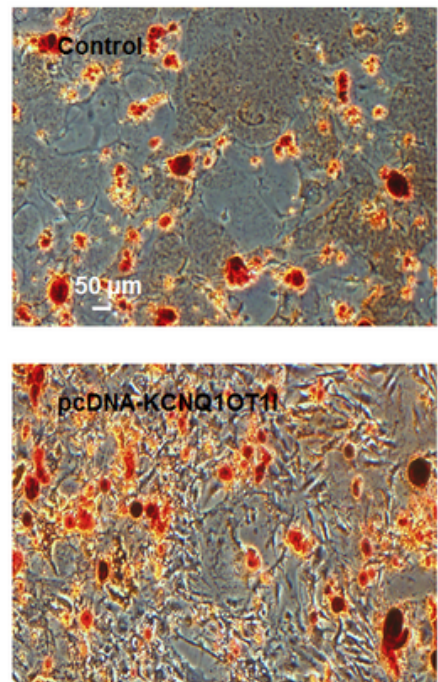

C
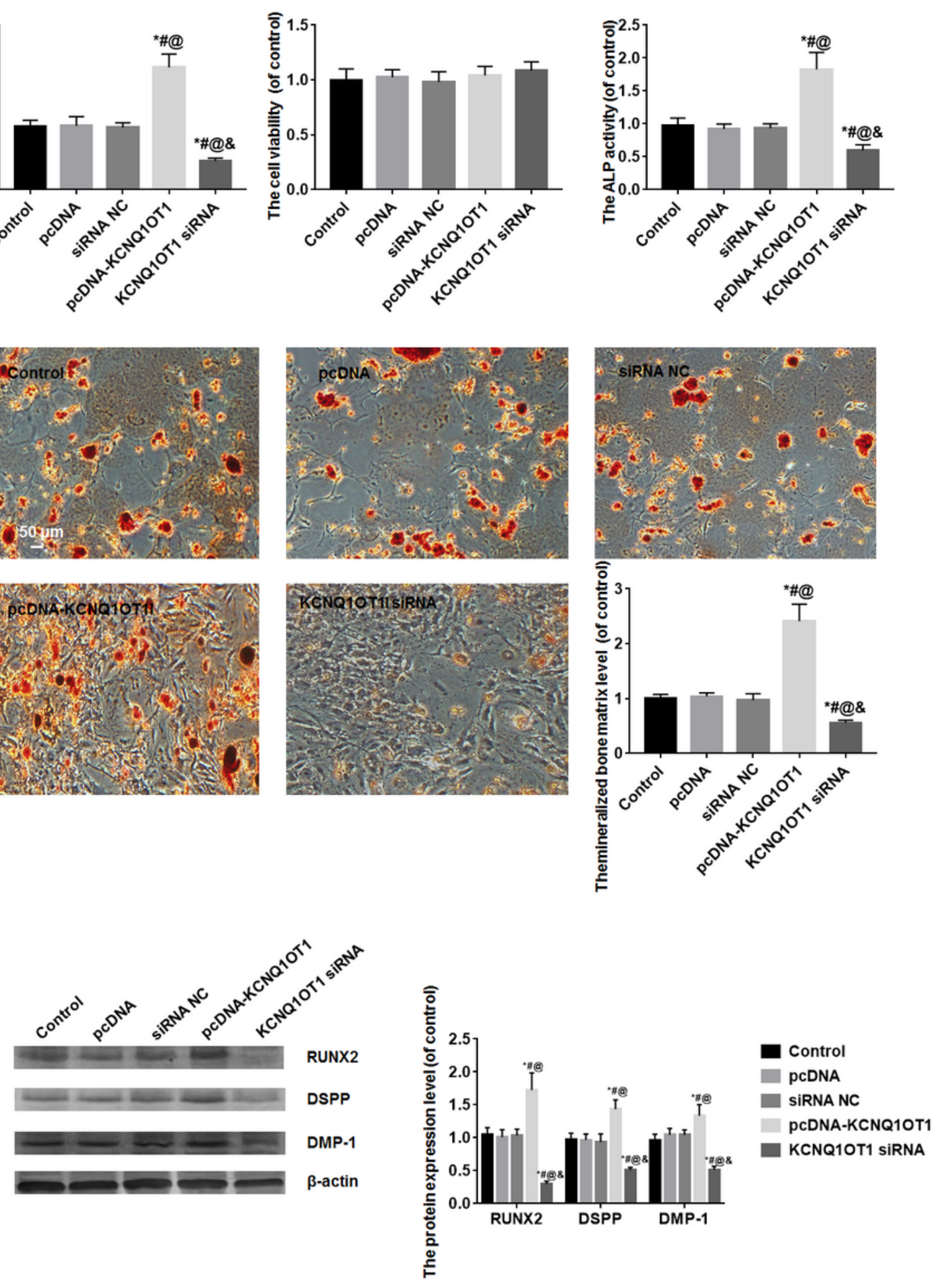

Figure 2

The effect of LncRNA-KCNQ10T1 on the cell viability and odontoblastic differentiation of DPSCs. (A) LncRNA-KCNQ10T1 expression level of DPSCs transfected with pcDNA-KCNQ10T1 significantly increased, and that of DPSCs transfected with KCNQ10T1 siRNA notably decreased. (B) The transfection did not affect the viability of DPSCs. (C) After 14 days of odontoblastic differentiation, ALP activity in the pcDNA-KCNQ10T1 group increased and in the KCNQ10T1 siRNA group decreased comparison with the 
control and NC groups. (D) After 14 days of odontoblastic differentiation, the mineralized bone matrix in the pcDNA-KCNQ10T1 group increased and in the KCNQ10T1 siRNA group decreased comparison with the control and NC groups. (E) After 14 days of odontoblastic differentiation, RUNX2, DSPP, and DMP-1 protein expression levels in the pCDNA-KCNQ10T1 group increased and in the KCNQ10T1 siRNA group decreased comparison with the control and NC groups. Bar $=50 \mu \mathrm{m}$. * vs. control group, $\mathrm{P}<0.05$; \# vs. pcDNA group, $\mathrm{P}<0.05$; @ vs. siRNA NC group, $\mathrm{P}<0.05$; \& vs. pcDNA-KCNQ10T1 group, $\mathrm{P}<0.05$.
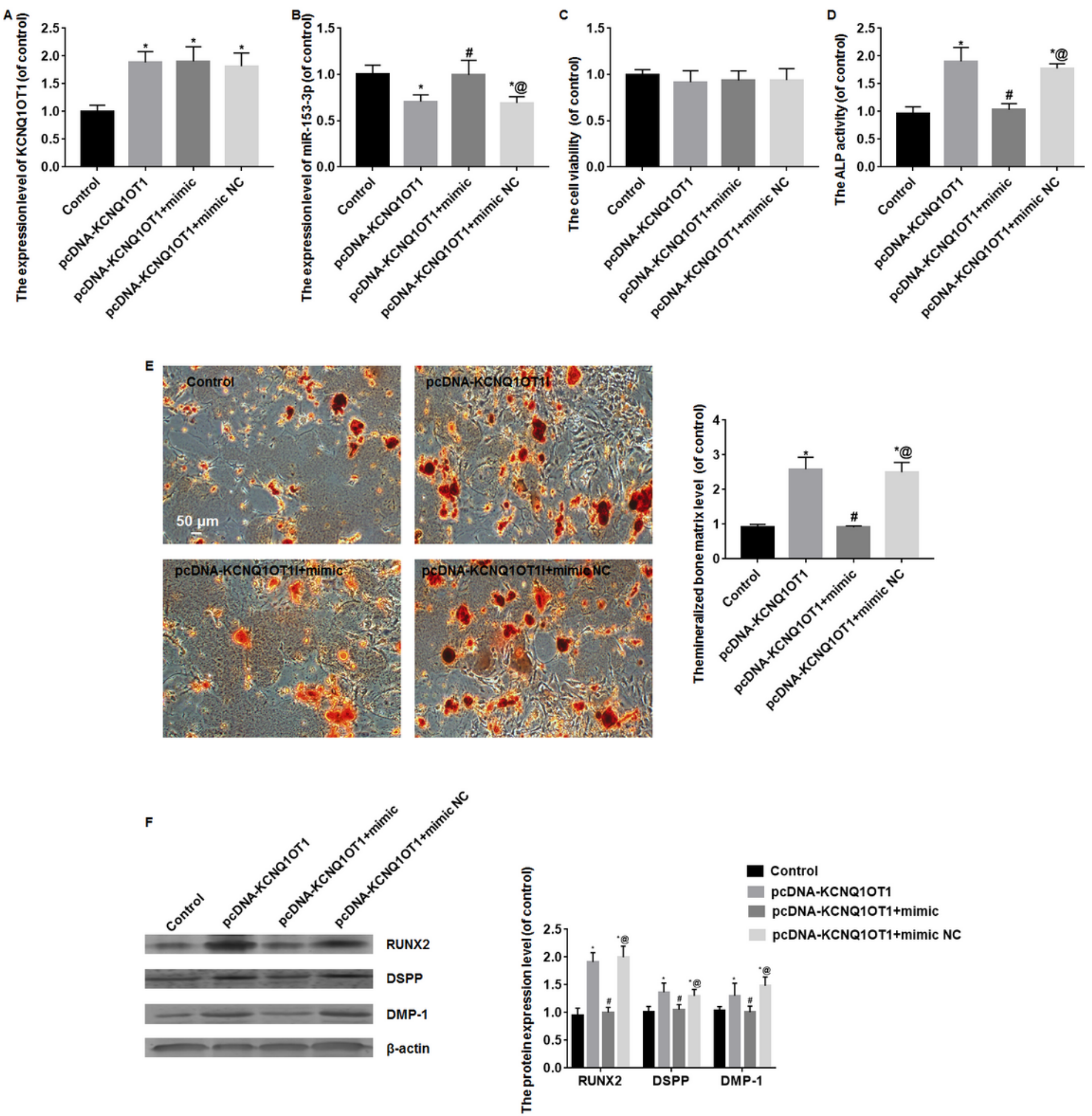

Figure 3 
Overexpression of miR-153-3p reverses the promotion of odontoblastic differentiation induced by LncRNA-KCNQ10T1. (A) LncRNA-KCNQ10T1 expression level of DPSCs transfected with pcDNAKCNQ10T1 or pcDNA-KCNQ10T1+mimic or pcDNA-KCNQ10T1+mimic NC significantly increased. (B) miR-153-3p expression level of DPSCs transfected pcDNA-KCNQ10T1 or pcDNA-KCNQ10T1+mimic NC significantly decreased, and miR-153-3p expression level of DPSCs transfected with pcDNA-

KCNQ10T1+mimic was similar to the control group. (C) The results of CCK-8 assay showed the transfection did not affect the viability of DPSCs. (D-F) After 14 days of odontoblastic differentiation, the ALP activity, mineralized bone matrix, RUNX2, DSPP, and DMP-1 protein expression levels in the pcDNAKCNQ10T1 group increased comparison with the control group. While DPSCs were co-transfected with pcDNA-KCNQ10T1 and miR-153-3p mimic the ALP activity, mineralized bone matrix, RUNX2, DSPP, and DMP-1 protein expression levels were close to the control group. Bar $=50 \mu \mathrm{m} .{ }^{*}$ vs. control group, $\mathrm{P}<$ 0.05; \# vs. pcDNA-KCNQ10T1 group, P < 0.05; @ vs. pcDNA-KCNQ10T1+mimic group, $\mathrm{P}<0.05$.

A
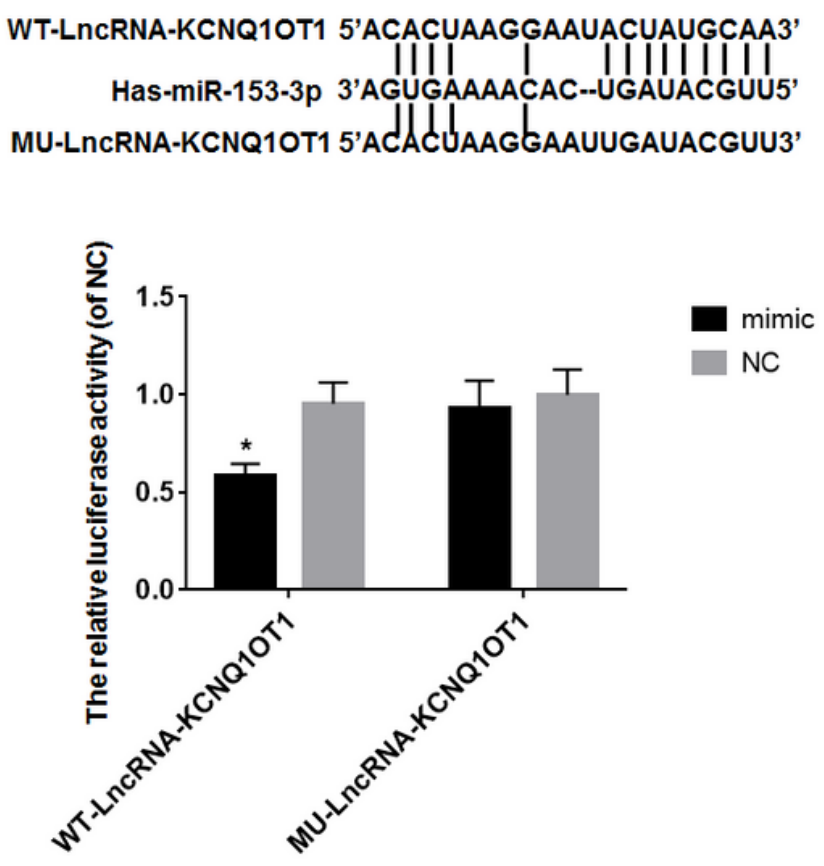

B

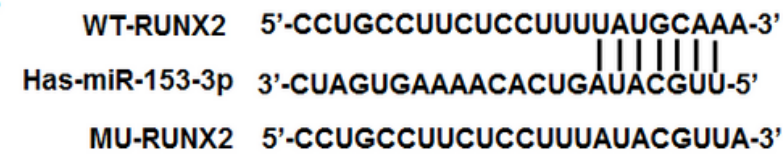

MU-RUNX2 5'-CCUGCCUUCUCCUUUAUACGUUA-3'

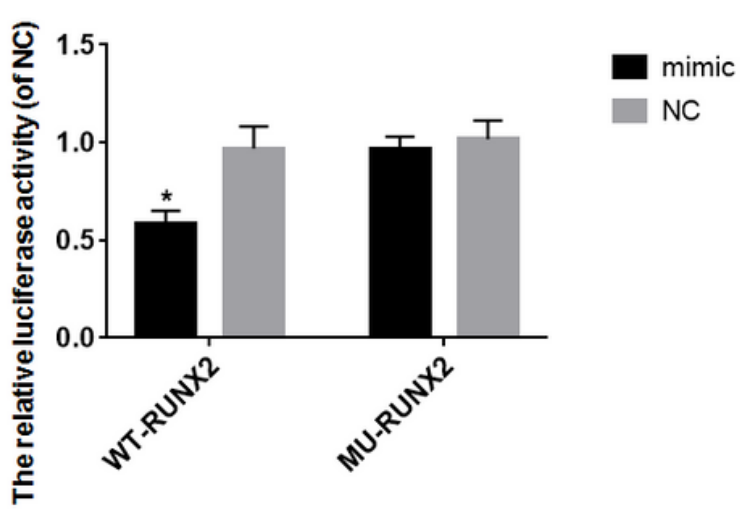

\section{Figure 4}

LncRNA-KCNQ10T1 act as a sponge of miR-153-3p and miR-153-3p target on RUNX2. (A) In the WTLncRNA-KCNQ10T1 reporter gene system, the relative activity of luciferase in mimic group was significantly lower than that in NC group. When the binding fragment of LncRNA-KCNQ10T1 was mutated, the luciferase relative activity of mimic group was similar to the NC group. (B) In the WT-RUNX2 reporter gene system, the relative activity of luciferase in mimic group was significantly lower than that in NC group. When the core sequence of 3'UTR of RUNX2 was mutated, the luciferase relative activity of mimic group was similar to the NC group. * vs. NC group, $\mathrm{P}<0.05$. 